\title{
Helicity of magnetic vortices and skyrmions in soft ferromagnetic nanodots and films biased by stray radial fields
}

\author{
R. V. Verba $\odot,{ }^{1,2, *}$ D. Navas $\odot,{ }^{1,3}$ S. A. Bunyaev, ${ }^{1}$ A. Hierro-Rodriguez,${ }^{1,4,5}$ K. Y. Guslienko,,${ }^{6,7}$ \\ B. A. Ivanov, ${ }^{2,8,9}$ and G. N. Kakazei ${ }^{1}$ \\ ${ }^{1}$ Institute of Physics for Advanced Materials, Nanotechnology and Photonics, Departamento de Fisica e Astronomia, \\ Universidade do Porto, 4169-007 Porto, Portugal \\ ${ }^{2}$ Institute of Magnetism, Kyiv 03142, Ukraine \\ ${ }^{3}$ Instituto de Ciencia de Materiales de Madrid, CSIC, 28049 Madrid, Spain \\ ${ }^{4}$ Departamento de Física, Universidad de Oviedo, 33007 Oviedo, Spain \\ ${ }^{5}$ Centro de Investigación en Nanomateriales y Nanotecnología, CINN (CSIC - Universidad de Oviedo), 33940 El Entrego, Spain \\ ${ }^{6}$ Departamento Física de Materiales, Universidad del País Vasco, EHU, 20018 San Sebastián, Spain \\ ${ }^{7}$ IKERBASQUE, The Basque Foundation for Science, 48013 Bilbao, Spain \\ ${ }^{8}$ Faculty of Radiophysics, Electronics and Computer Systems, Taras Shevchenko National University of Kyiv, Kyiv 01601, Ukraine \\ ${ }^{9}$ National University of Science and Technology “MISiS," Moscow 119049, Russian Federation
}

(Received 31 October 2019; revised manuscript received 31 January 2020; accepted 11 February 2020; published 27 February 2020)

\begin{abstract}
Static magnetization configurations of thin soft ferromagnetic films and nanodots, coupled to a hard antidot matrix with out-of-plane magnetization, are studied by micromagnetic simulations and analytical calculations. When the antidot matrix produces sufficient stray fields, having radial symmetry, these nanostructures support the formation of topologically nontrivial magnetic configurations-vortices and skyrmions in nanodots and films, respectively. It is demonstrated that the studied nanostructure reveals an additional degree of freedom-the helicity of the vortex or skyrmion - which can be tuned on demand by a variation of the material parameters and geometry. The variation of helicity $\gamma$ is not abrupt. In addition to Neel-like (radial) vortices and skyrmions $(\gamma=0, \pi)$, it is possible to achieve unconventional configurations with an intermediate helicity $\gamma \neq 0, \pm \pi / 2, \pi$, which transform to common Bloch-like configurations $(\gamma= \pm \pi / 2)$ in the limit of negligible stray fields from the matrix. We present an analytical model, which allows us to calculate the stability region of pure Neel-like states, outside which unconventional magnetization states with intermediate helicity are realized.
\end{abstract}

DOI: 10.1103/PhysRevB.101.064429

\section{INTRODUCTION}

Magnetic nanostructures can reveal a considerable variety of different static magnetization configurations. Control of static magnetic states opens a route to significantly modify dynamical properties of nanostructures, such as response to quasistatic and microwave magnetic fields, spectrum and spatial profiles of spin-wave modes, interaction with spin-polarized or pure spin current, etc. Among other magnetic configurations, a great attention is paid to topologically nontrivial magnetization states, like skyrmions [1-3], vortices [4,5], chiral domain walls [6,7], magnetic bobbers [8], Bloch points [9-11], Hopfions [12,13], and others. Magnetic skyrmions were recently proposed for application in future generation of magnetic memory devices $[3,14,15]$. Magnetic vortices, although being intensively studied for the two past decades, still attract a significant research interest in relation with applications in spin-transfer-torque devices [16-18].

Here we consider two-dimensional nontrivial magnetization textures, the most prominent examples of which are vortices and skyrmions. The main characteristic of a two-dimensional magnetization texture is the $\pi_{2}$-topological

*Corresponding author: verrv@ukr.net charge $[19,20]$, also often called the "skyrmion number," which counts how many times magnetization distribution wraps the unit sphere. In addition to the topological charge, other characteristics of two-dimensional magnetization textures, namely, the polarity, vorticity, and helicity, are widely used $[21,22]$. The easiest way to understand these characteristics is to consider a circularly symmetric magnetization texture centered in the coordinate origin $(r=0)$, use polar coordinate system $\boldsymbol{r}=(r, \chi)$, and express magnetization in terms of the spherical polar and azimuthal angles $\theta(r)$ and $\phi(r, \chi)$ as $\boldsymbol{M} / M_{s}=(\sin \theta \cos \phi, \sin \theta \sin \phi, \cos \theta)$. Then, polarity is simply the direction of the magnetization in the texture center, $p=M_{z}(r=0) / M_{s}=\cos \theta(r=0)$. The azimuthal angle of magnetization can be expressed as $\phi(r, \chi)=v \chi+\gamma(r)$. Here $v$ is the vorticity (also called "the winding number"), which defines how many times magnetization rotates in the texture plane when passing one turn around the texture core. For vortices and skyrmions the vorticity is usually equal to $v=1$. Finally, $\gamma$ is the helicity, which determines the angle between the radial direction and in-plane part of magnetization, as depicted in Fig. 1(a). More general definitions can be found in the Appendix.

In most previous works only magnetization textures with certain helicities have been studied. Indeed, a magnetic vortex 


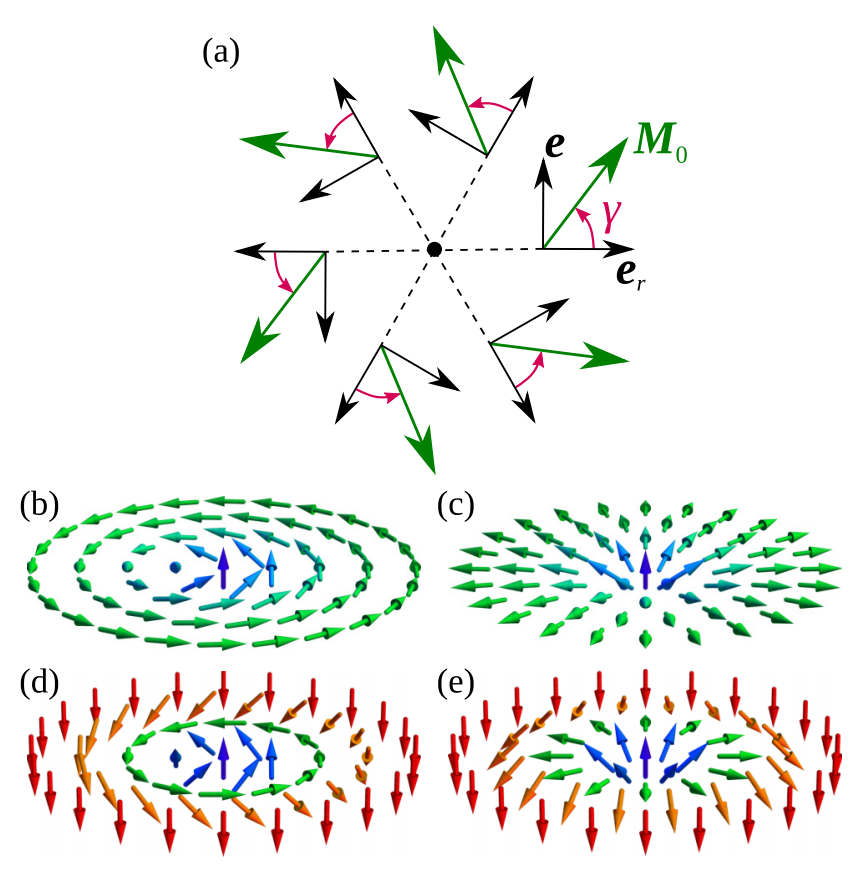

FIG. 1. (a) Schematic picture of a magnetic topological soliton state showing the definition of the helicity $\gamma$. Examples of commonly studied two-dimensional magnetization textures are the (b) common (Bloch) vortex (helicity $\gamma=\pi / 2)$, (c) radial vortex $(\gamma=0)$, (d) Bloch skyrmion $(\gamma=\pi / 2)$, and (e) Neel skyrmion $(\gamma=0)$.

in a circular magnetic nanodot has closed-flux Bloch-like structure, which minimizes its demagnetization energy [4,23]. An example of such a vortex is shown in Fig. 1(b). For Bloch-like vortices the helicity is constant and is equal to $\gamma= \pm \pi / 2$, where the sign determines the sense of rotation of magnetization, clockwise or counterclockwise. Radial vortices [Fig. 1(c)], for which $\gamma=0, \pi$, can be stabilized by the interfacial Dzyaloshinskii-Moriya interaction (IDMI) [24,25], or in vertical stacks of nanodots with antiferromagnetic interlayer exchange coupling [26,27]. Other vortices having $\gamma \neq$ $0, \pm \pi / 2, \pi$ are often called "unconventional vortices" [28]. Such kind of vortices can be stabilized by the introduction of specific boundary conditions, like a connection to magnetic nanowires [28]. Also, an unconventional structure of a vortex is realized in thick magnetic dots, but it was found that in this case the vortex magnetization configuration and, consequently, its helicity are thickness dependent [28-30].

The situation with skyrmions is similar. IDMI in ultrathin films favors the formation of Neel skyrmions characterized by $\gamma=0, \pi$ [Fig. 1(e)] [31-33]. Bulk Dzyaloshinskii-Moriya interaction in B20 compounds [34,35], as well as dipolar interaction in magnetic dots having perpendicular anisotropy and no DMI [36,37], lead to the stabilization of Bloch skyrmions [Fig. 1(d)]. Skyrmions with other helicities were predicted in systems with comparable bulk and interfacial DMI [38]. Also, they were observed in multilayer structures with IDMI in which, however, they are realized only in a few layers of the multilayer stack, while common Neel skyrmions exists in the rest of layers [39-41]. There is only one observation of such "unconventional" skyrmions in single layers, which was achieved by a delicate tuning of IDMI with electric field [42].
Recently, we have proposed an alternative method for the stabilization of magnetic skyrmions and vortices [43,44]. It is based on the exploiting of the radial magnetic field, which could be created, e.g., by an antidot matrix. This approach allows one to stabilize magnetic skyrmions and their nontopological counterparts in soft ferromagnetic films having neither DMI nor perpendicular magnetic anisotropy, just by dipolar and interlayer exchange coupling to a perpendicularly magnetized hard antidot matrix [44]. Simultaneously, in the application to soft magnetic nanodots, radial stray fields from an antidot matrix allow for significant reduction of the minimal size and thickness of dots, where magnetic vortices can be stabilized in Ref. [43].

In addition, the radial stray fields can stabilize magnetization configurations with arbitrary helicity $\gamma$. In this paper we systematically study the variation of helicity of magnetic vortices and skyrmions stabilized by dipolar fields of radial symmetry. It will be shown that, by the variation of geometry and material parameters, it is possible to vary the helicity on demand, achieving, thus, an additional degree of freedom of topological magnetization textures.

The paper is organized as follows. In Sec. II we introduce magnetic nanostructures, which can be used for the stabilization of magnetic skyrmions and vortices by dipolar stray fields, and present examples of magnetization textures with arbitrary helicity, which are realized in these nanostructures. Also, we discuss why the helicity matters, how it can affect properties of the magnetization state, and how it can be detected experimentally. Then, in Sec. III we present an analytical model, which sheds light on the nature of unconventional states with intermediate helicities (not Bloch or Neel ones) and allows us to calculate the boundary of stability of pure Neel (radial) states, beyond which unconventional magnetization states are realized. Phase diagrams of magnetic vortices and skyrmions, showing the regions of parameters where different states are realized, with emphasis on their helicity, are presented and discussed in Sec. IV. Finally, conclusions are given in Sec. V.

\section{NANOSTRUCTURES UNDER STUDY AND UNCONVENTIONAL MAGNETIZATION TEXTURES}

The main idea of the stabilization of magnetic skyrmions and unconventional vortices in soft magnetic films and dots is the application of magnetic fields having radial symmetry $[43,44]$. Here we describe one possible approach, which, with some modifications, allows us to achieve stabilization of both vortices and skyrmions.

The basic nanostructure for the enhancement of the stability of magnetic vortices is shown in Fig. 2(a). It is a layer of hard magnetic material with out-of-plane magnetization and the thickness $t_{\mathrm{HL}}$, having an antidot (a hole) of the diameter $d_{\mathrm{ad}}$. Inside the antidot there is a soft magnetic nanodot of the thickness $t_{\mathrm{d}}$ and diameter $d_{\mathrm{d}}$. The dot is grown on the same substrate as the hard magnetic layer, resulting in asymmetric vertical position of the dot respective to the hard layer. We assume that the direct exchange coupling between the dot and hard layer is absent in this nanostructure, that is the case, e.g., if the dot diameter is smaller than the antidot diameter, as it is shown in Fig. 2(a). The main impact of the hard layer is 


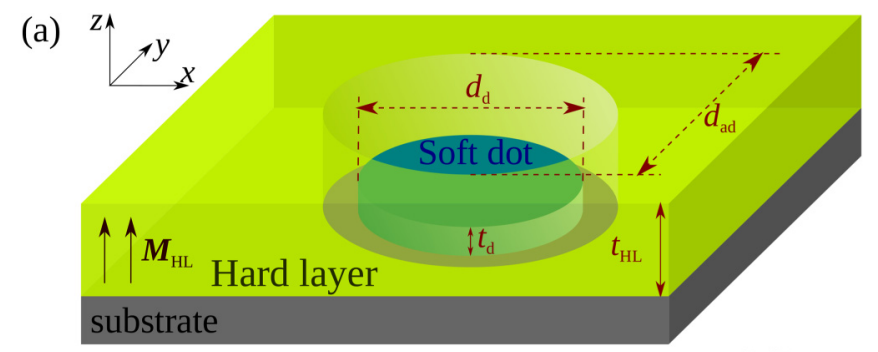

(b)
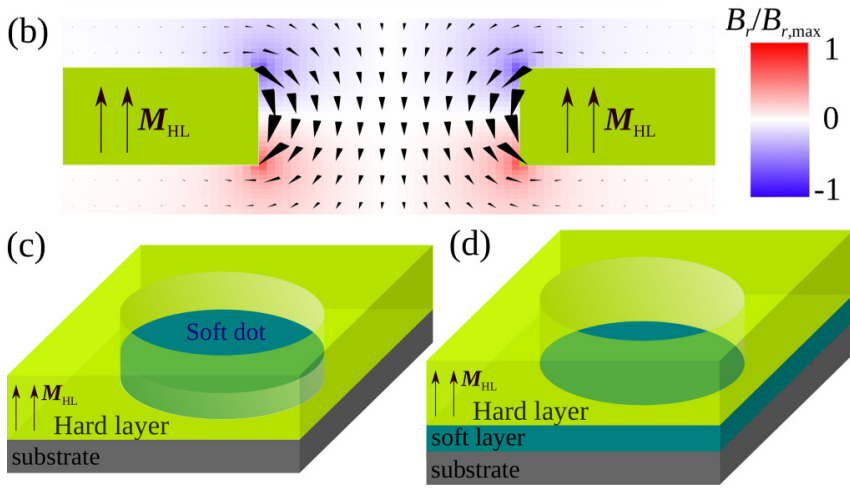

FIG. 2. (a) A sketch of the patterned nanostructure, in which a soft magnetic nanodot is biased by the stray fields produced by an antidot (hole) in a hard magnetic layer with out-of-plane magnetization. (b) Distribution of the stray fields in the antidot; arrow length corresponds to the field magnitude; color corresponds to the field radial component $B_{r}$. In this example, the maximum of the radial field component is $B_{r, \max } \approx 0.5 \mathrm{~T}\left(t_{\mathrm{HL}}=30 \mathrm{~nm}, d_{\mathrm{ad}}=\right.$ $80 \mathrm{~nm}, M_{\mathrm{HL}}=10^{6} \mathrm{~A} / \mathrm{m}$ ). Panel (c) shows the modification of the nanostructure, in which a soft disk is in direct contact with a hard layer. (d) Nanostructure for the stabilization of skyrmions, where a soft magnetic film is exchange coupled to the antidot matrix.

the creation of magnetostatic stray fields inside the antidot and in its vicinity. The distribution of these fields is shown in Fig. 2(b). It is clear that the stray fields are radially symmetric and have radial $B_{r}$ and perpendicular $B_{z}$ components. The radial component is zero only at the central plane and reaches a maximum close to the top and bottom surfaces of the hard layer (see color scale). That is why it is important to place the dot close to the top or bottom of the hard layer.

In addition to the nanostructure shown in Fig. 2(a), we study a similar structure in which the dot and antidot diameters are the same, so that the dot is exchange coupled to the antidot matrix at its lateral edges [Fig. 2(c)]. Practical realization of the cases of presence or absence of the exchange coupling depends on the fabrication technology. As it will be shown below, this coupling at the lateral edges has an evident effect on the helicity of the magnetic vortices.

Finally, by placing a continuous soft magnetic film underneath a hard layer with an antidot [Fig. 2(d)], we create the nanostructure, in which magnetic skyrmions and nontopological solitons can be stabilized [44]. In this structure, the interlayer exchange coupling ensures that the magnetization of the soft layer is perpendicular to the plane in the area of direct contact to the hard layer (except for a small area near the antidot edge). Thus, it allows us to realize magnetization textures with opposite or same out-of-plane magnetizations in the core and at the periphery, i.e., skyrmions and their nontopological counterparts. Simultaneously, we note that the magnetic nanostructures, shown in Figs. 2(c) and 2(d), are almost the same from the point of the helicity, as it is shown below in Sec. IV.

As we have already noted, the structures shown in Fig. 2 are not unique for the stabilization of magnetic skyrmions in soft magnetic layers and enhancement of the vortex stability in small soft magnetic dots. The key ingredient for the stabilization of these magnetization textures is the strong radial component of the stray field of the matrix. Such kind of fields can be, obviously, created in other ways. For example, the stray fields of radial symmetry can be created by the tip of a magnetic force microscope, resulting in the observation of unconventional vortices in soft magnetic dots, which, however, disappear as soon as the tip is removed [45-47].

The magnetization configurations in all the nanostructures were studied using the MUMAX3 micromagnetic solver [48]. As a soft magnetic material (dot or film) we choose permalloy $\mathrm{Ni}_{80} \mathrm{Fe}_{20}$, having the saturation magnetization of $M_{s}=8.1 \times 10^{5} \mathrm{~A} / \mathrm{m}$ and the exchange stiffness $A=1.05 \times$ $10^{-11} \mathrm{~J} / \mathrm{m}$. Parameters of the hard magnetic layer were set as $M_{s}=10^{6} \mathrm{~A} / \mathrm{m}, A=2 \times 10^{-11} \mathrm{~J} / \mathrm{m}$, and the uniaxial outof-plane magnetic anisotropy constant $K_{u}=7 \times 10^{5} \mathrm{~J} / \mathrm{m}^{3}$ for the structures with soft magnetic dots [Figs. 2(a) and 2(c)] and $K_{u}=9 \times 10^{5} \mathrm{~J} / \mathrm{m}^{3}$ for the structure with continuous soft film [Fig. 2(d)]. These parameters correspond to FePd, FePt, and CoPt multilayers of different composition [49,50]. Strictly speaking, the main important characteristic of the hard layer for the studied case is the value of $M_{s}$, because the stray fields created by the hard layer are proportional to $M_{s}$. The out-of-plane anisotropy should be simply strong enough to guarantee a stable out-of-plane magnetization state of the hard layer in zero external magnetic field. For the case of exchange-coupled soft magnetic film, the anisotropy of the hard layer should overcome demagnetization energy of both hard and soft films, and the uniaxial anisotropy constant was chosen larger (see details in Ref. [44]). The minimal required anisotropy constant, in a general case, depends on the antidot lattice geometry [51,52]. Our simulations have confirmed stable quasiuniform out-of-plane magnetization of the hard layer in zero bias magnetic field (after the magnetization saturation in the out-of-plane direction) in the whole studied range for chosen material parameters. The variation of magnetic state diagrams with a variation of material parameters is discussed in Sec. III. The cell size in simulations was chosen to be $2.5 \times 2.5 \times 1.5 \mathrm{~nm}^{3}$ for antidots having diameters larger than $70 \mathrm{~nm}$ and $1.25 \times 1.25 \times 1 \mathrm{~nm}^{3}$ for smaller antidots. To avoid edge effects we set periodic boundary conditions with the period of $400 \mathrm{~nm}$, which is large enough to avoid any interdot interaction. Below we look at the magnetization configurations, which are realized at zero external field after it gradual decreases from the perpendicular saturation (in the $+z$ direction).

Magnetization configuration of the soft magnetic dot (layer) depends on the geometry of both the dot (layer) and the antidot matrix. One can achieve a quasisingle domain state, a vortex (skyrmion) state, or more complex magnetization configurations by varying the geometric parameters $[43,44]$. In Fig. 3 we present several examples of possible vortex states, which were observed at remanence in the nanostructure 

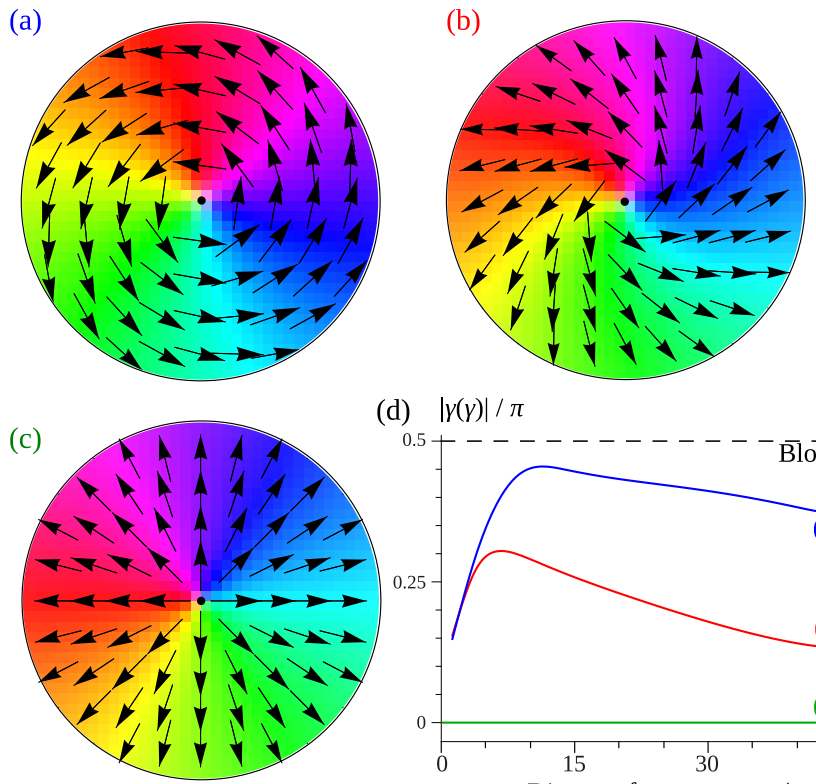

(d) $|\gamma(\gamma)| / \pi$

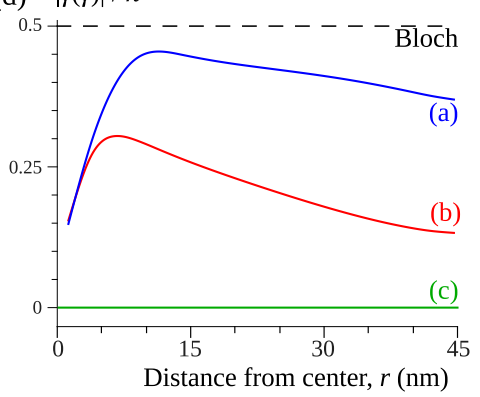

FIG. 3. Remanent magnetization configuration of the 90-nmdiameter dot without direct contact to the antidot matrix [nanostructure shown in Fig. 2(a)]: (a) unconventional vortex having the averaged helicity $\bar{\gamma}=0.43 \pi$ which is realized at the hard matrix thickness $t_{\mathrm{HL}}=9 \mathrm{~nm}$, (b) unconventional vortex with $\bar{\gamma}=0.2 \pi$ $\left(t_{\mathrm{HL}}=21 \mathrm{~nm}\right)$, and (c) radial vortex $\left(t_{\mathrm{HL}}=34.5 \mathrm{~nm}\right)$; dot thickness in all the panels is $t_{\mathrm{d}}=3 \mathrm{~nm}$. (d) Position-dependent helicity $\gamma(r)$ of the vortices, shown in the panels (a)-(c); the dashed line shows the limiting case of a Bloch vortex.

[Fig. 2(a)] having different thickness of the antidot matrix. A matrix of a large thickness produces the radial stray fields, which are strong enough to stabilize the radial vortex state [Fig. 3(c)] having the in-plane magnetization component aligned to the stray field direction. When decreasing the matrix thickness the vortex magnetization becomes more and more curled, and the vortices become intermediate between the radial and Bloch vortices, as shown in Figs. 3(a) and 3(b). A noticeable feature of these unconventional vortices is that the angle between the in-plane magnetization and the radial direction is not the same everywhere within the dot. In other words, the helicity of these vortices is position dependent, $\gamma=\gamma(r)$. It has a maximum near the vortex core and decreases towards the dot edges, as shown in Fig. 3(d). We will characterize such textures by the averaged helicity $\bar{\gamma}$, defined in the Appendix [Eq. (A4)].

It should be emphasized that we do not observe a pure Bloch vortex with the helicity $\gamma= \pm \pi / 2$ in the presence of the antidot matrix until it creates nonzero radial stray fields in the dot (the averaged radial component disappears only if the dot is placed at the central plane of the matrix). We note also that the magnetization configurations with clockwise and counterclockwise rotation are degenerated in the magnetic energy and the realization of one of them is a random process; i.e., the vortices with helicities $\gamma(r)$ and $-\gamma(r)$ can appear with equal probability in the considered case. If the static magnetization of the matrix is reversed, $\boldsymbol{M}_{\mathrm{HL}}=-M_{\mathrm{HL}} \boldsymbol{e}_{z}$, the radial vortex will have inward structure $(\gamma=\pi)$, while the
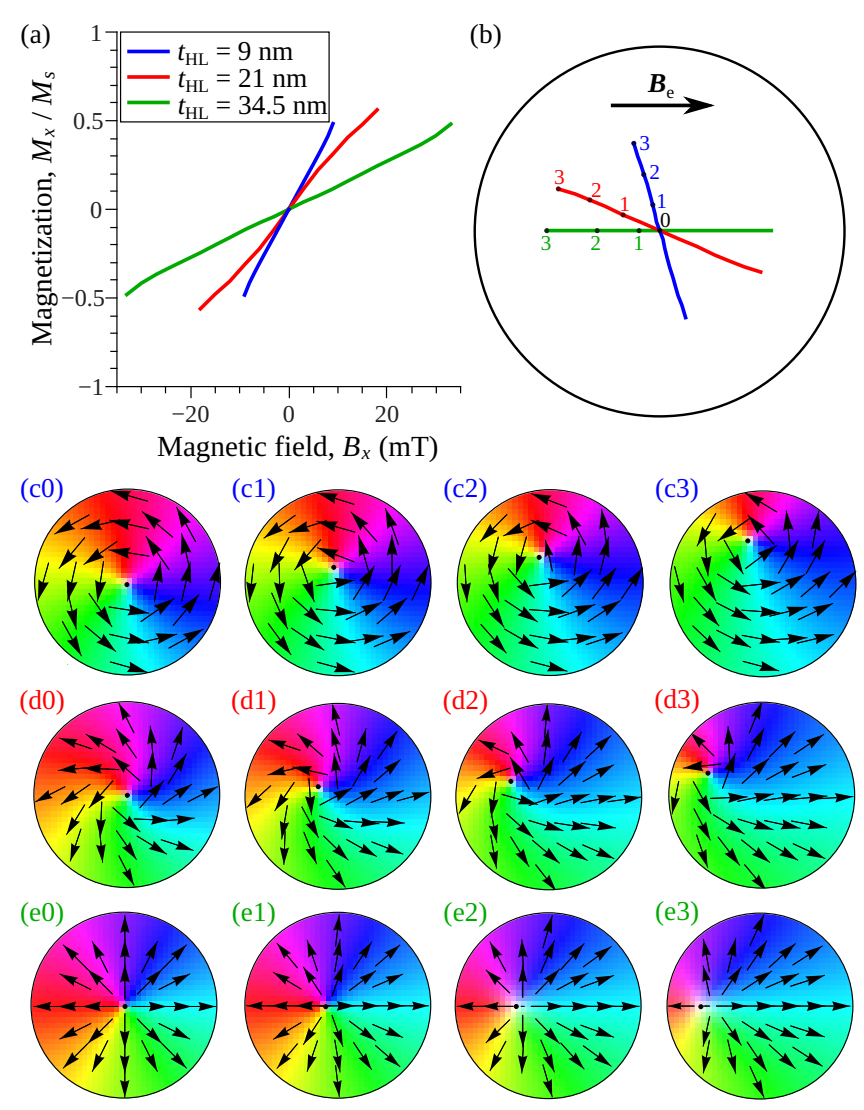

FIG. 4. The displacement of vortices with different helicity under applied in-plane magnetic field: (a) minor hysteresis loops (without reaching the vortex annihilation) and (b) trajectories of the vortex core; the circle denotes the dot boundary, and panels (c)-(e) correspond to magnetization distributions of a dot under applied field; numbers $0-3$ denote the point at the trajectories, shown in panel (b). Dot thickness $t_{\mathrm{d}}=3 \mathrm{~nm}$, diameter $d_{\mathrm{d}}=90 \mathrm{~nm}$, the hard layer thickness $t_{\mathrm{HL}}=9 \mathrm{~nm}(\mathrm{c}), t_{\mathrm{HL}}=21 \mathrm{~nm}(\mathrm{~d})$, and $t_{\mathrm{HL}}=34.5 \mathrm{~nm}$; corresponding averaged helicity of vortices is $\bar{\gamma}=0.43 \pi$ (c), $\bar{\gamma}=$ $0.2 \pi(\mathrm{d})$, and $\bar{\gamma}=0$ (e).

degenerated unconventional vortices will be characterized by the helicities $\gamma(r)$ and $[\pi-\gamma(r)]$, respectively.

It should be noted that $\gamma$ is not an artificial parameter, characterizing magnetic vortices and other textures. Different helicity results in a different response to an external force, a different spectrum of spin-wave modes, etc. Just as an illustration in Fig. 4 we present trajectories of the vortex cores under applied in-plane external magnetic field and corresponding minor hysteresis loops (i.e., we do not reach the vortex annihilation field; features of the vortex annihilation and nucleation in such nanostructures are described in Ref. [43]). It is known that a common Bloch vortex moves in the perpendicular to the applied field direction in the dot plane [4]. From Fig. 4 one can see that, in contrast, the radial vortex moves parallel to the field, while unconventional vortices move at a certain angle to the field direction and the larger the averaged helicity of the vortex the larger this angle. This behavior can be naturally understood recalling that the vortex movement is governed by the Zeeman energy, which promotes a larger part of the vortex to have magnetization aligned 
(a)

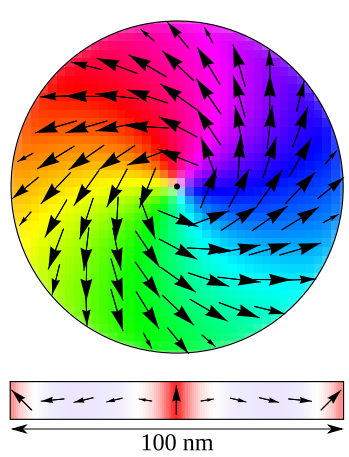

(b)

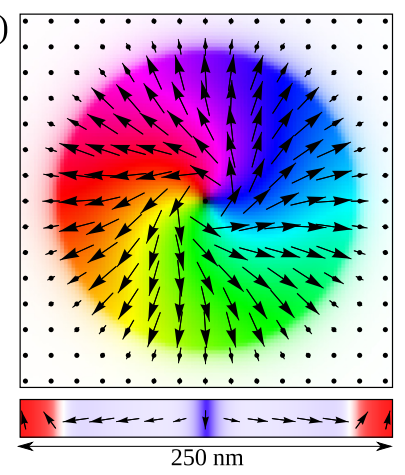

FIG. 5. Example magnetization configurations with an intermediate helicity, which are realized in the nanostructures shown in Figs. 2(c) and 2(d): (a) magnetic vortex with $\tilde{\gamma}=0.28 \pi$ in the soft dot having lateral contact with a hard matrix $\left(d_{\mathrm{d}}=100 \mathrm{~nm}, t_{\mathrm{d}}=\right.$ $3 \mathrm{~nm}, t_{\mathrm{HL}}=9 \mathrm{~nm}$ ) and (b) magnetic skyrmion having $\tilde{\gamma}=0.08 \pi$ in the soft film, exchange coupled to the hard matrix $\left(d_{\mathrm{ad}}=200 \mathrm{~nm}\right.$, soft layer thickness $t_{\mathrm{SL}}=3 \mathrm{~nm}, t_{\mathrm{HL}}=21 \mathrm{~nm}$ ). Top, in-plane view; bottom, $x-z$ cross section.

to the field direction. Importantly, the vortex trajectories are almost straight lines, although unconventional vortices have position-dependent helicity. This means that the angle of the vortex motion is determined by the averaged helicity. Additionally, this displacement of a vortex under applied magnetic field could be used for the experimental characterization of the vortex helicity.

The response of a magnetic vortex and other magnetic textures to an in-plane external field is not the only process affected by the helicity. For example, it was shown in Ref. [53] that interaction of magnetic skyrmions with spin current also depends on the helicity, resulting in helicity-dependent motion of skyrmions driven by the spin current. Also, if using a vortex or skyrmion as magnetization configuration of the fixed layer in a spin-transfer-torque oscillator, the distribution of spin-polarized current becomes naturally dependent of their helicity. This spin-polarized current can create different torques affecting the magnetization of the free layer as it was shown using an example of a free layer in the skyrmion state [54]. Finally, one can expect that the helicity of magnetization textures affects the spin-wave spectrum and spatial profiles of spin-wave modes, as well as nonlinear magnetization oscillations [55].

Similar unconventional magnetic configurations with a position-dependent intermediate helicity were observed in other nanostructures, shown in Fig. 2. The presence of exchange interaction with the matrix at the lateral edges of a soft magnetic dot [Fig. 2(c)] creates a certain out-of-plane tilt of the dot magnetization near these edges [Fig. 5(a)], while the in-plane magnetization component resembles the unconventional vortices shown in Fig. 3. In a soft magnetic film coupled to a hard antidot matrix, in a certain range of parameters, we observe unconventional magnetic skyrmions, the structure of which is characterized by position-dependent helicity [Fig. 5(b)]. Full diagrams of magnetization configurations of the studied nanostructures, showing the regions of realization of magnetization textures with intermediate helicities, are shown below in Fig. 6.

Thus, all considered nanostructures demonstrate the same qualitative features. First, in a certain range of the magnetic and geometric parameters pure radial (Neel) configuration can be realized. Second, pure Bloch states are not observed until a soft magnetic dot (film) is biased by radial stray fields. And third, the helicity is position dependent in unconventional textures with intermediate helicities.

\section{ANALYTICAL MODEL}

In order to explain the observed features we developed an analytical model. We consider magnetic energy of a soft magnetic dot as a functional of its magnetization, analysis of which allows us to find possible stationary solutions (magnetization configurations) and analyze stability of these solutions. In this section only the case of a soft magnetic dot, with or without contact to the matrix at the lateral edges, is considered. Regarding the problem of the magnetization texture helicity, the case of magnetic skyrmions in a soft magnetic film is almost equivalent to the case of a dot with exchange coupling at the lateral edges. In the model we consider only the magnetization of the soft magnetic dot, assuming that the magnetization in the hard layer is fixed-it is uniform and it is directed perpendicularly to the layer plane, $\boldsymbol{M}_{\mathrm{HL}}=p M_{\mathrm{HL}} \boldsymbol{e}_{z}$, where $p= \pm 1$ corresponds to the magnetization direction "up" or "down," $M_{\mathrm{HL}}$ is the saturation magnetization of the hard layer, and $\boldsymbol{e}_{z}$ is the out-of-plane unit vector. This assumption is valid if the out-of-plane magnetic anisotropy of the hard layer is sufficiently strong. The hard layer is considered as continuous uniform ferromagnetic-although some of the hard materials are, in fact, multilayers (e.g., FePt), for the calculation of stray fields produced by the antidot these details do not matter. In addition, we assume that the thickness of a soft magnetic dot is of the order of or smaller than the material exchange length, so that the magnetization distribution of the soft layer can be considered uniform along the thickness $z$ coordinate. Finally, we neglect the difference between radii of the dot and antidot in the case of absence of direct contact between them, setting $R_{\mathrm{d}}=R_{\mathrm{ad}}=R$.

The magnetic energy of a soft magnetic dot consists of the exchange, Zeeman, and dipolar contributions:

$$
W[\boldsymbol{M}(\boldsymbol{r})]=W_{\mathrm{ex}}+W_{\mathrm{Z}}+W_{\mathrm{dip}} .
$$

For the derivation of explicit expressions of each contribution, it is convenient to describe magnetization of the dot in terms of polar and azimuthal angles, $\boldsymbol{M}(\boldsymbol{r})=$ $M_{\mathrm{d}}[\sin \theta \cos \phi, \sin \theta \sin \phi, \cos \theta]$, which both depend on twodimensional radius vector $\boldsymbol{r}$ in the dot plane, $\theta=\theta(\boldsymbol{r}), \phi=$ $\phi(r) . M_{\mathrm{d}}$ here is the saturation magnetization of the soft dot. Then, the energy of the nonuniform exchange is derived as

$$
W_{\mathrm{ex}}=t_{\mathrm{d}} \frac{\mu_{0} M_{\mathrm{d}}^{2} \lambda^{2}}{2} \int\left[(\nabla \theta)^{2}+(\nabla \phi)^{2} \sin ^{2} \theta\right] d \boldsymbol{r},
$$

where $\lambda$ is the exchange length of the dot material.

The term $W_{Z}$ corresponds to the energy of the dot magnetization in an external magnetic field. In our case this total magnetic field, acting on the dot, consists of the stray fields, produced by the antidot matrix, and the external magnetic field itself. Stray fields of the antidot have two components, radial $B_{r}$ and perpendicular $B_{z}$ [see Fig. 2(b)]. The latter is 
approximately uniform within the antidot, except for a small area near the antidot boundaries (see Ref. [44]), and can be compensated by an external perpendicular field. In the following we consider the situation when the total out-ofplane magnetic field acting on the dot is absent, that simplifies further consideration. Simultaneously, we note that the presence of a nonzero total perpendicular field (e.g., if external field is zero) insignificantly affects the helicity of magnetic vortices, as was confirmed by micromagnetic simulations.

The radial component of the antidot stray fields can be expressed as $B_{r}=p \mu_{0} M_{\mathrm{HL}} f_{\beta}(r) / 2$. In the approximation of an infinitely thin soft ferromagnetic layer, placed exactly at the bottom of the matrix, the dimensionless function is

$$
f_{\beta}(r)=\left.\frac{2}{\pi k} \sqrt{\frac{R}{r}}\left[K(k)\left(1-\frac{k^{2}}{2}\right)-E(k)\right]\right|_{z=0} ^{z=\tilde{t}_{\mathrm{HL}}},
$$

where $k^{2}=4 r R /\left[(r+R)^{2}+z^{2}\right]$, and $E(k)$ and $K(k)$ are complete elliptic integrals (details of the calculation of the stray fields can be found in supplementary materials of Ref. [44]). In the range $r \ll R$ this expression can be approximated as

$$
f_{\beta}(r)=\frac{r}{2 R}\left[1-\left(1+\beta^{2}\right)^{-3 / 2}\right]
$$

where $\beta=\tilde{t}_{\mathrm{HL}} / R$ is the antidot aspect ratio. Thus, the radial component of the stray fields increases linearly with increasing of the distance from the antidot center. The value of $B_{r}$ increases faster approaching the antidot boundary and reaches a maximum at $r=R$. A finite thickness of the soft magnetic layer leads to modifications of Eq. (3) (an explicit expression cannot be derived in this general case); however, until $t_{\mathrm{SL}} \ll$ $t_{\mathrm{HL}}$, the difference is small and can be neglected.

In the case of a dot placed within the antidot as shown in Fig. 2(a), the same expressions (3) and (4) can be applied. However, a part of the hard layer produces zero radial stray fields, averaged over the dot thickness, since it is located at the same vertical position as the dot. This effect can be approximately accounted for by the introduction of the "effective thickness" $\tilde{t}_{\mathrm{HL}}$ of the hard layer. For thin dots, when $t_{\mathrm{d}} \ll t_{\mathrm{HL}}$, the best approximation is $\tilde{t}_{\mathrm{HL}}=t_{\mathrm{HL}}-t_{\mathrm{d}}$. Naturally, in the case of a soft magnetic film placed underneath the matrix [Fig. 2(d)] no effective thickness should be introduced and $\tilde{t}_{\mathrm{LH}}=t_{\mathrm{HL}}$. The same relation is valid, e.g., if an antidot is filled by a nonmagnetic material of the thickness equal to the hard layer thickness, and a dot is placed on top of it.

Using the above expressions for the radial stray fields of the antidot, the dot Zeeman energy is expressed as

$$
W_{\mathrm{H}}=-p t_{\mathrm{d}} \frac{\mu_{0} M_{\mathrm{d}} M_{\mathrm{HL}}}{2} \int f_{\beta}(r) \sin \theta \cos [\phi-\chi] d \boldsymbol{r} .
$$

The dipolar energy, in a general case, is a nonlocal functional of the magnetization, and can be expressed via tensorial magnetostatic Green's function [56] $\hat{\boldsymbol{G}}\left(\boldsymbol{r}, \boldsymbol{r}^{\prime}\right)$ as

$$
W_{\mathrm{dip}}=-t_{\mathrm{d}} \frac{\mu_{0} M_{\mathrm{d}}^{2}}{2} \int d \boldsymbol{r} \int d \boldsymbol{r}^{\prime} \boldsymbol{m}(\boldsymbol{r}) \cdot \hat{\boldsymbol{G}}\left(\boldsymbol{r}, \boldsymbol{r}^{\prime}\right) \cdot \boldsymbol{m}\left(\boldsymbol{r}^{\prime}\right),
$$

where $\boldsymbol{m}(\boldsymbol{r})=\boldsymbol{M}(\boldsymbol{r}) / M_{\mathrm{d}}$ is the normalized magnetization distribution of a dot. Usage of this general expression, however, does not allow us to perform further qualitative and quantitative analysis. Therefore, we use the following approximation.
In a general case, the dipolar energy can be divided into two parts, related to the surface magnetic charges $\boldsymbol{M} \cdot \boldsymbol{n}$, where $\boldsymbol{n}$ is the unit vector normal to the surface, and contribution from the volume magnetic charges $(\nabla \cdot \boldsymbol{M})$. The contribution from the surface charges at the dot top and bottom surfaces can be approximated simply as

$$
W_{\mathrm{dip}, \mathrm{s}}=t_{\mathrm{d}} \frac{\mu_{0} M_{\mathrm{d}}^{2}}{2} \int \cos ^{2} \theta d \boldsymbol{r},
$$

which describes the in-plane shape anisotropy of a thin dot. This local approximation of the part of the magnetostatic energy is valid for thin dots with aspect ratio $t_{\mathrm{d}} / R \ll 1$, and characteristic sizes of magnetization textures larger than the dot thickness. In our case of dots with thickness less than the material exchange length these conditions are satisfied. For the calculation of the contribution from the volume charges and surface charges at the dot lateral edges we assume that the solutions we are searching for are radially symmetric, i.e., $\theta=\theta(r)$ and $\phi=\chi+\gamma(r)$. This is a natural assumption because of radial symmetry of the nanostructure. Then, using explicit expressions for the Green's function in a polar coordinate system [57], one can find that the magnetostatic energy is determined only by the radial magnetization component $m_{r}$. This can be easily understood because in the circularly symmetric case the component $m_{\chi}$ creates neither volume charges $\left(\nabla \cdot \boldsymbol{M} \sim \partial_{r}\left[r m_{r}\right]\right)$ nor surface charges at the lateral edges $\boldsymbol{M} \cdot \boldsymbol{e}_{r}$. Thus, the second part of the dipolar energy can be written as

$$
W_{\mathrm{dip}, \mathrm{v}}=-t_{\mathrm{d}} \frac{M_{\mathrm{d}}}{2} \int \sin \theta \cos [\phi-\chi] B_{\mathrm{dip}, r} d \boldsymbol{r},
$$

where

$$
B_{\mathrm{dip}, r}(\boldsymbol{r})=\mu_{0} M_{\mathrm{d}} \int G_{r r}\left(\boldsymbol{r}, \boldsymbol{r}^{\prime}\right) \sin \theta\left(r^{\prime}\right) \cos \left[\phi\left(\boldsymbol{r}^{\prime}\right)-\chi^{\prime}\right] d \boldsymbol{r}^{\prime}
$$

is the radial component of dipolar (demagnetization) fields of the dot, and $G_{r r}$ is the $r r$ component of the tensorial magnetostatic Green's function [57].

Possible magnetization configurations of the dot correspond to local or global minima of the energy functional $W[\boldsymbol{M}(\boldsymbol{r})]$. Magnetization configurations which minimize the energy functional can be found as solutions of the corresponding Euler-Lagrange equations. In this paper we are looking only at the function $\phi(r, \chi)$, the Euler-Lagrange equation for which yields

$$
\begin{aligned}
\lambda^{2} \nabla\left(\sin ^{2} \theta \nabla \phi\right)= & \left(\frac{p M_{\mathrm{HL}}}{2 M_{\mathrm{d}}} f_{\beta}(r)+\frac{B_{\mathrm{dip}, r}[\theta, \phi]}{\mu_{0} M_{\mathrm{d}}}\right) \\
& \times \sin \theta \sin (\phi-\chi) .
\end{aligned}
$$

It is clear that this equation has simple solutions $\phi=\chi$ and $\phi=\chi+\pi$, which correspond to the radial vortex. Simultaneously, the function $\phi=\chi \pm \pi / 2$, which describes a Bloch vortex, is not a solution of Eq. (10). Moreover, Eq. (10) has no solutions with a constant helicity, $\phi=\chi+\gamma$, except for the radial vortex solution. All other possible solutions are characterized by position-dependent helicity $\gamma=\gamma(r)$. That is exactly what was observed in the simulations.

The reason for such behavior is the presence of radial stray fields created by the antidot matrix. As soon as it disappears 
$\left[M_{\mathrm{HL}}=0\right.$ in Eq. (10)], the Bloch vortex becomes a solution, since demagnetization field $B_{\mathrm{dip}, r}=0$ for this solution (moreover, the Bloch vortex is the only stable solution among circularly symmetric ones in this case). The observation of radial and unconventional vortices, but not Bloch vortices in the presence of radial stray fields, is analogous to the behavior of a single-domain thin magnetic dot in an outof-plane magnetic field. Indeed, the magnetostatic energy is quadratic in magnetization components and acts similarly to shape or material anisotropy, defining "easy direction" $\boldsymbol{m}=$ $\pm \boldsymbol{e}_{\chi}$. Radial stray fields are perpendicular to this direction. In this case it is known that a sufficient external magnetic field can align magnetization in its direction, but any small field tilts magnetization from the easy-plane direction. In our case, a sufficient radial stray field can stabilize the radial vortex, but the Bloch vortex ("easy-axis direction") cannot be realized until the stray fields are removed completely.

In the following we formulate a criterion, when a radial vortex is achieved, and when an unconventional vortex with position-dependent helicity is observed. This can be done by the finding of the boundary of stability of the radial vortex respective to the curling of magnetization. Of course, it could happen that in a certain range of parameters both radial and unconventional vortices are metastable, and the realization of one of them depends on the magnetic prehistory of the nanostructure. In our method, when the vortices are achieved after gradual decrease of external magnetic field starting from out-of-plane saturation, the formation of radial vortices is favored at the initial stage of the field decrease. Therefore, if the radial vortex is stable, we always observe it. Unconventional vortices are observed if the radial one becomes unstable. Also, we will see below (Sec. IV) that the helicity changes gradually near the boundary of radial vortex stability, meaning that the bistability region, if it exists, is small.

Usage of a general expression for the magnetostatic energy (8) is not straightforward due to its nonlocal nature. Instead, we model the effect of demagnetization by the approximate local term

$$
W_{\mathrm{dip}, \mathrm{v}}=t_{\mathrm{d}} \frac{\mu_{0} M_{\mathrm{d}}^{2}}{2} D \int \sin ^{2} \theta \cos ^{2}(\phi-\chi) d \boldsymbol{r} .
$$

This term describes an "effective anisotropy" in the azimuthal $\chi$ direction. Similarly to the exact expressions Eqs. (8) and (9), this term is a square function of $\sin \theta$ and $\cos (\phi-\chi)$. In the limiting case $\theta=\pi / 2$ and $\phi-\chi=\gamma=$ const the exact expression coincides with the approximate one that allows us to determine the coefficient $D$. Within the range of small dot aspect ratios $t_{\mathrm{d}} / R \ll 1$ it is approximately valid, $D \approx 2 t_{\mathrm{d}} / R$.

We also assume that the polar magnetization angle distribution $\theta(r)$ does not change with the transition from the radial to the unconventional vortex solution, i.e., that the vortex core remains of the same size. It is a natural assumption, as the function $\theta(r)$ is mainly determined by competing of the in-plane shape anisotropy and exchange energy, which are commonly larger than the Zeeman energy of stray fields and the nonlocal part of the magnetostatic energy.

Solutions corresponding to the radial vortices are $\phi=\chi$ and $\phi=\chi+\pi$. Although they are both stationary solutions of Eq. (10), it is clear that for the magnetization of the matrix in the up direction, $p=+1$, only the first solution could be stable, while for opposite matrix magnetization $p=-1$ only the solution $\phi=\chi+\pi$ is stable. A general solution for the unconventional vortex can be written as $\phi=\chi+\gamma(r)$ or $\phi=\chi+\pi+\gamma(r)$, respectively. As we consider the problem of radial vortex stability respective to small perturbations, we set $|\gamma(r)| \ll 1$. Also, it is convenient to introduce a new variable $\mu(r)=\gamma(r) \sin \theta(r)$ that is useful for the determination of boundary conditions (see below). It is clear that the stabilities of the solutions $\mu=0$ and $\gamma=0$ are equivalent (at the points where $\sin \theta=0$ the parameter $\gamma$ cannot be determined).

Finally, we introduce dimensionless coordinate $\xi=r / R$. With all the above defined assumptions we can reduce the energy functional $W[\boldsymbol{M}]$ to the normalized functional of the function $\mu(\xi)$ :

$$
\tilde{W}[\mu(\xi)]=\int_{0}^{1} \Phi(\mu) d \xi,
$$

where

$$
\begin{aligned}
\Phi(\mu)= & {\left[\frac{1}{2}\left(\mu^{\prime}\right)^{2}-\mu \mu^{\prime} \cot \theta \theta^{\prime}+\frac{1}{2} \mu^{2} \cot ^{2} \theta\left(\theta^{\prime}\right)^{2}\right.} \\
& \left.+\left(\frac{A}{2 \sin \theta} \tilde{f}_{\beta}(\xi)-B\right) \mu^{2}\right] \xi .
\end{aligned}
$$

Short notation $\mu^{\prime}=d \mu / d \xi$ stands here for the derivative; the coefficients

$$
A=\frac{M_{\mathrm{HL}} R^{2}}{2 M_{\mathrm{d}} \lambda^{2}}\left(1-\frac{1}{\left(1+\beta^{2}\right)^{3 / 2}}\right), \quad B=\frac{2 t_{\mathrm{d}} R}{\lambda^{2}}
$$

describe normalized strength of the Zeeman and magnetostatic energy, respectively; and the function

$$
\tilde{f}_{\beta}(\xi)=f_{\beta}(\xi R)\left(1-\frac{1}{\left(1+\beta^{2}\right)^{3 / 2}}\right)^{-1}
$$

gives the normalized profile of the radial stray fields produced by the antidot. The approximation $\tilde{f}_{\beta}(\xi) \approx \xi / 2$ is valid in the range of small $\xi$ [see Eq. (4)].

Boundary conditions for the function $\mu(\xi)$ are clear. In the dot center $\mu(0)=0$. Conditions at the dot lateral edge depend on the presence or absence of direct contact to the matrix. In the latter case a free boundary condition is realized, $\mu^{\prime}(1)=0$. In the former case, exchange coupling to the matrix fixes the dot magnetization at the lateral edge to the direction equal or close to the matrix magnetization direction, so we can set $\mu(1)=0$. In contrast to the function $\mu(\xi)$, boundary conditions for $\gamma(\xi)$ are not so clear, since it is simply undefined for out-of-plane magnetization, which takes place at the dot center and, in the case of contact to the matrix, at the lateral edges. We note that in the nanostructure with continuous soft magnetic film [Fig. 2(d)] one should use the strong pinning boundary condition $\mu(1)=0$, since interlayer exchange coupling fixes the film magnetization outside the antidot. Of course, there could be a certain distance between the area of perpendicular magnetization and the antidot edge (domain-wall width), but it is small and affects insignificantly the results presented below. 
The Euler-Lagrange equation for the functional (12) is derived as

$$
\mu^{\prime \prime}+\frac{\mu^{\prime}}{\xi}=\left(\frac{A \tilde{f}_{\beta}}{\sin \theta}-2 B-\left(\theta^{\prime}\right)^{2}+\frac{1}{\xi} \cot \theta \theta^{\prime}+\cot \theta \theta^{\prime \prime}\right) \mu .
$$

It has stationary solution $\mu=0$ for both fixed and free boundary conditions at $\xi=1$, which describes the radial vortex (Neel skyrmion). This stationary solution corresponds to a minimum of the functional, if two conditions, Legendre's and Jacobi's, are satisfied [58]. Legendre's condition requires that $\partial^{2} \Phi / \partial \mu^{\prime 2}>0$, and is always satisfied in our case $\left(\partial^{2} \Phi / \partial \mu^{\prime 2}=\xi\right)$. The second condition considers solutions of Jacobi's equation for the functional $W[\mu]$. Since the functional (13) is a quadratic form of $\mu$ and $\mu^{\prime}$ and we consider the stability of the stationary state $\mu=0$, Jacobi's equation, which describes the second variation of the functional, is the same as the Euler-Lagrange equation (16). Jacobi's condition is satisfied if this equation, accompanied by the boundary conditions for the function $\mu$, has no nontrivial $[\mu(\xi) \neq 0]$ solutions for any $\tilde{\xi} \in(0,1]$. This condition is equivalent to the condition of absence of solutions with negative eigenvalues (unstable solutions) at the interval $\xi \in[0,1]$.

Equation (16) contains the function $\theta(\xi)$, which is unknown. However, the last term, containing the derivative $\theta^{\prime}$, is local and acts only in the core region and, in the case of a dot in contact with the matrix, close to the dot lateral edges. Similarly, a difference between the function $(1 / \sin \theta)$ and 1 exists only in these small regions. Moreover, it could be shown that these local terms act oppositely: The first term destabilizes the stationary solution $\mu=0$, while the second one stabilizes it. Therefore, it would not produce a large error if one disregards these local terms and simplifies Eq. (16) to

$$
\mu^{\prime \prime}+\mu^{\prime} / \xi-\left(A \tilde{f}_{\beta}(\xi)-B\right) \mu=0 .
$$

Unfortunately, Eq. (17) cannot be solved analytically even using the approximation $\tilde{f}_{\beta}(\xi) \approx \xi / 2$. Instead, we solve it numerically, and find the relation between the parameters $A=\tilde{A}(B, \beta)$, at which Jacobi's condition starts to fails, i.e., at which Jacobi's equation has a nontrivial solution for $\tilde{\xi}=1$. For the fixed boundary condition at the dot lateral edge, this function, found numerically, is nicely fitted by the dependence

$$
\tilde{A} \approx 0.8(B-6.3) \sqrt{B-C_{\beta}},
$$

where

$$
C_{\beta}=-15+18 \exp \left[-1.5 \beta^{2}\right] .
$$

Returning to the initial material and geometrical parameters, the condition of radial vortex stability appears as

$$
\frac{M_{\mathrm{HL}} R^{2}}{M_{\mathrm{d}} \lambda^{2}}\left(1-\frac{1}{\left(1+\beta^{2}\right)^{3 / 2}}\right)>1.6\left(\frac{2 t_{\mathrm{d}} R}{\lambda^{2}}-6.3\right) \sqrt{\frac{2 t_{\mathrm{d}} R}{\lambda^{2}}-C_{\beta}} .
$$

If this condition is not satisfied, the radial vortex is unstable and an unconventional vortex state with intermediate helicity is realized.

From this equation we can analyze what factors lead to the stabilization or destabilization of the radial vortex state. First is the ratio $M_{\mathrm{HL}} / M_{\mathrm{d}}$, higher values of which lead to a more stable radial vortex state. This ratio, in fact, describes the relative strength of the radial stray fields created by the matrix in comparison to the internal fields in the dot, in particular, effective demagnetization fields. The second stabilization factor of the radial vortex is a decrease of the dot thickness, since demagnetization fields, acting contrary to the radial state, are proportional to the dot thickness-to-radius aspect ratio [see Eq. (11)]. A smaller ratio $R / \lambda$ (smaller dots or larger material exchange length) is also a stabilization factor of the radial vortex state. This is due to a position-dependent helicity of the intermediate vortex state creating an additional increase of the exchange energy, and the energy increases with $R$ decreasing. Finally, the dependence on the antidot aspect ratio $\beta$ is not so evident from Eq. (20). The term in the left-hand side of the condition describes increased stability of the radial vortex at larger $\beta$, because it corresponds to larger antidot stray fields. Simultaneously, at a fixed value of $M_{\mathrm{HL}}\left[1-\left(1+\beta^{2}\right)^{-3 / 2}\right]$, which corresponds to a fixed strength of the radial stray fields close to the dot center, a decrease of $\beta$ stabilizes the radial vortex state. This happens because the radial fields of the antidot with small aspect ratio $\beta$ have stronger "tails" close to the antidot boundaries, while the stray fields of antidots with $\beta \gtrsim 2$ are much closer to the approximation Eq. (4).

Unfortunately, in the case of free boundary conditions at the dot lateral edge we were unable to find such a simple relation as Eq. (18). Therefore, in the next section we use the function $\tilde{A}(B, \beta)$, which describes a boundary of Jacobi's condition fulfillment, extracted from numerical solution of Jacobi's equation. However, for a sufficiently thick hard matrix, when the antidot aspect ratio $\beta>2$ and has no effect on the stability condition, the following simple condition of the vortex state stability can be derived:

$$
A>\tilde{A} \approx 0.8 B \sqrt{B+5} .
$$

For the case $B \gg 1$, which corresponds to thick and/or large radius dots, this condition becomes almost the same as in the case of fixed boundary conditions. This happens because of the large demagnetization energy for such dots, in comparison to which the effect of the exchange at the lateral dot edges becomes less important. Simultaneously, in the opposite case of smaller dots (not large parameter $B$ ), the strength of radial stray fields, required for the stabilization of the radial vortex, is larger for the free boundary conditions than for the fixed one (larger value of $\tilde{A}$ at given $B$ ). In other words, the presence of exchange coupling to the matrix at the lateral edges increases the stability of the radial vortex, and this effect is more pronounced for smaller and thinner dots. The reason for this additional stabilization is that exchange coupling to the matrix fixes the dot magnetization at the lateral edges and, thus, suppresses a gain for a curled vortex state coming from the contribution to the demagnetization energy from surface charges at the edges.

\section{TRANSITION OF HELICITY OF VORTICES AND SKYRMIONS}

In this section we present and discuss full diagrams of remanent states of the studied nanostructures, obtained from micromagnetic simulations. First, we fix the thickness of the soft dot or layer to $t_{\mathrm{d}}=t_{\mathrm{SL}}=3 \mathrm{~nm}$ and vary the thickness of the hard layer and diameter of the antidot. In the case of a 


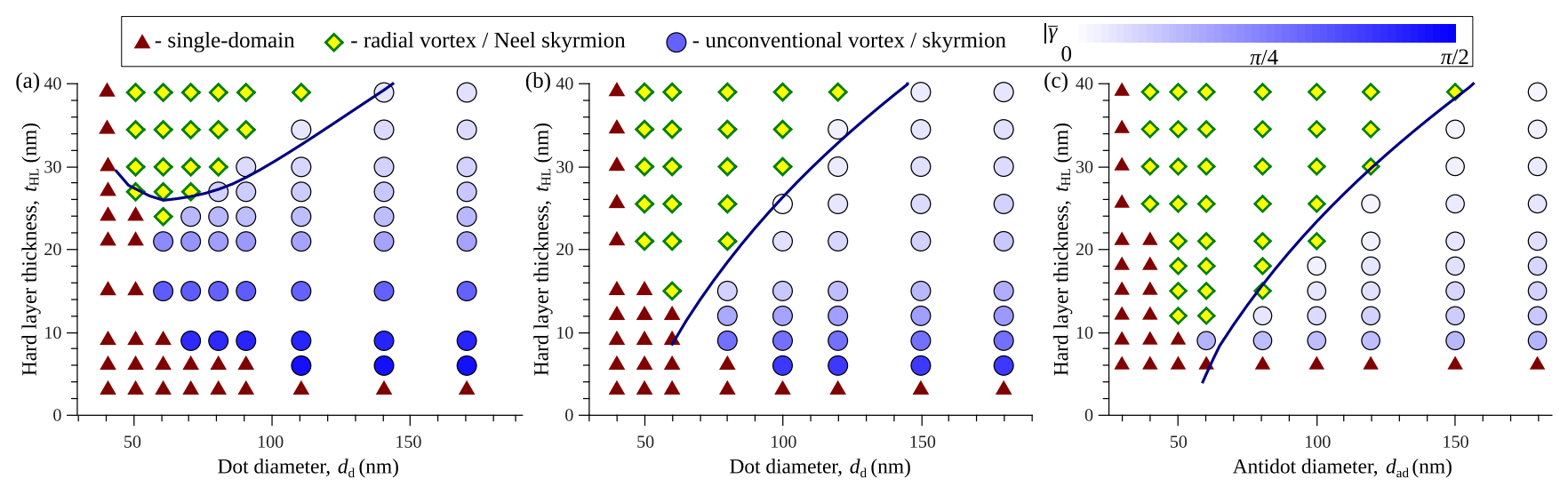

FIG. 6. Diagrams of remanent states, showing regions of the realization of the single-domain quasiuniform state, radial vortex or Neel skyrmions, and unconventional vortices and skyrmions, the helicity of which is shown by color scale; solid lines represent analytically calculated boundaries of the Neel-like state stability. (a) Soft magnetic dot without exchange coupling to the matrix [structure shown in Fig. 2(a)]. (b) Soft magnetic dot with exchange coupling at the lateral edges [Fig. 2(c)]. (c) Soft ferromagnetic film, coupled to the hard layer by exchange interaction [Fig. 2(d)]. Thickness of the dot and soft layer $t_{\mathrm{d}}=t_{\mathrm{SL}}=3 \mathrm{~nm}$.

soft magnetic dot without contact to the matrix [nanostructure Fig. 2(a)], the diameter of the dot is $10 \mathrm{~nm}$ smaller than the antidot diameter.

Micromagnetic diagrams are shown in Fig. 6. For the smallest diameter antidots or thin hard layers the remanent state of the soft magnetic dot or layer is a quasi-single-domain state. It is a consequence of insufficient stray fields to stabilize the vortex or skyrmion in such nanostructures $[43,44]$. The minimal dot diameter, in which the vortex state is observed, is $50 \mathrm{~nm}$ for both cases of presence or absence of exchange coupling to the matrix. Interestingly, in the case of soft magnetic film coupled to the antidot matrix, the minimal antidot diameter for skyrmion stabilization is smaller; it is $40 \mathrm{~nm}$. This happens because the skyrmion is not localized exactly under the antidot. Magnetization of the soft layer changes also in a small region outside the antidot that reduces the exchange energy of essentially nonuniform magnetization distribution due to a larger "effective size" of the magnetization variation.

Outside the quasi-single-domain region the remanent state of a soft magnetic dot or film is a vortex or skyrmion, respectively. In full agreement with theoretical prediction, in the case of a sufficiently thick matrix and small diameter dots (antidots) we observe the radial vortex and Neel skyrmions, and a thicker matrix supports formation of a Neel-like state in larger dots (antidots). Unconventional vortices and skyrmions with intermediate helicities are observed within the last region of parameters. Importantly, the helicity of the vortex (skyrmion) changes gradually with geometrical parameters (see color scale in Fig. 6). It is small, $|\bar{\gamma}| \ll 1$, near the boundary of the Neel-like state stability and approaches $|\bar{\gamma}| \rightarrow \pi / 2$, but never reaches the pure Bloch state $|\gamma|=\pi / 2$ in the presence of the matrix. Thus, by choice of the geometrical and material parameters one can achieve the vortex or skyrmion state with an arbitrary desirable helicity.

One can conclude from Fig. 6 that the averaged helicity of skyrmions, realized in a soft magnetic film, is smaller compared to the helicity of vortices in a dot with exchange coupling to the matrix at the lateral edges [compare panels (b) and (c)]. This is another consequence of the fact that magnetization of the soft magnetic layer is not exactly out of plane in a certain area outside the antidot. In this area radial stray fields are the most strong (the maximum is at the antidot edge), forcing the in-plane projection of the film magnetization to have radial direction. This "edge region" contributes to the averaged helicity of skyrmions [see definition Eq. (A4)], making its value lower.

In Fig. 6 we also show the boundary of stability of the radial vortex (Neel skyrmion) obtained from the analytical model. One can see very good description of simulations data by the model. In particular, simulations show an evident enlargement of the stability range of the radial vortex state due to the presence of exchange coupling to the matrix at small dot diameters, in a full agreement with theoretical predictions. Simultaneously, we can note that the effect of exchange coupling to the matrix on the boundary between the vortex and single-domain state is much less pronounced. Thus, one can use the developed model to predict properties of nanostructures made of other materials. For example, it is clear that using the hard layer made of a ferromagnet with lower magnetization requires larger thickness of the antidot matrix for the stabilization of the radial vortex (Neel skyrmion). Simultaneously, the shift of the boundary between these states is not governed by the simple relation $M_{\mathrm{HL}}[1-$ $\left.\left(1+\beta^{2}\right)^{-3 / 2}\right]=$ const, which describes scaling of the radial stray fields in the antidot center [see Eq. (4)], because at low aspect ratios of antidots the "tails" of stray fields at the periphery are also important [coefficient $C_{\beta}$ in Eq. (20)].

Next we consider the effect of the dot thickness. As was obtained within the developed analytical model, a decrease of the dot thickness should favor the formation of the radial vortex state, since the demagnetization energy, acting contrary to the radial vortex stability decreases with the dot thickness faster than Zeeman energy of magnetization in radial stray fields, proportional to $t_{\mathrm{d}}^{2}$ and $t_{\mathrm{d}}$, respectively [see Eqs. (5) and (11)]. That is exactly what micromagnetic simulations show, as demonstrated in the diagram of states at fixed hard layer thickness in Fig. 7. One can note that the dependence of critical dot thickness for the radial to unconventional vortex transition on the dot radius is quite weak, somewhat more pronounced in the case of exchange coupling to the matrix. 


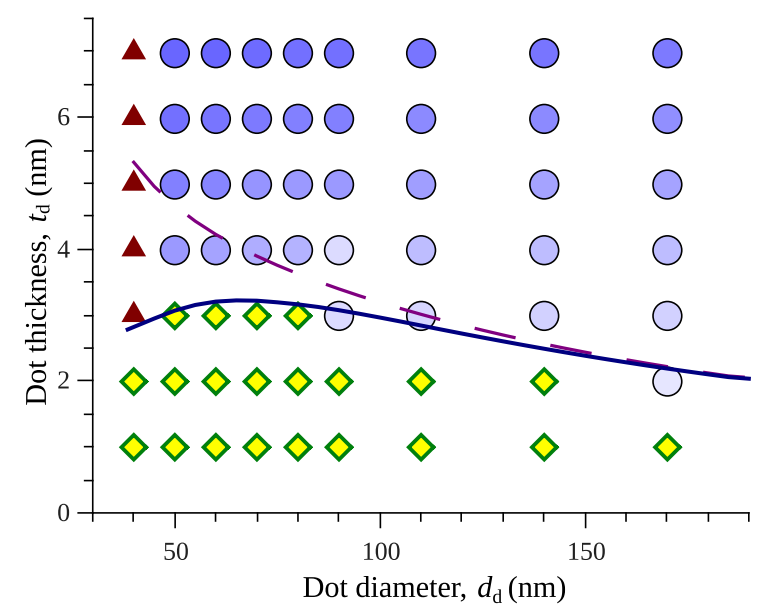

FIG. 7. Diagrams of remanent states of a soft magnetic dot without contact to the antidot matrix, showing the dependence on the dot thickness. Symbols, micromagnetic simulations; solid line, analytical calculation of the boundary between the radial and intermediate vortex states; dashed line, analytical calculations assuming the presence of the direct exchange contact of the dot and matrix. Thickness of hard layer $t_{\mathrm{HL}}=30 \mathrm{~nm}$. Notation of symbols is the same as in Fig. 6.

In the case of a soft magnetic film underneath the hard matrix the variation of the skyrmion helicity is the same. The only issue one should be aware of is that the presented model is valid if the interlayer exchange interaction is strong enough to guarantee out-of-plane magnetization direction of the soft magnetic layer away from the antidot. At a certain film thickness, depending on the interlayer exchange strength, this condition breaks, and instead of skyrmions more complex magnetization textures are realized. For the studied materials this critical thickness is about $5 \mathrm{~nm}$ [44]. Additionally, from Fig. 7 one can conclude that the smaller dot thickness allows for the stabilization of vortices in the dots with smaller diameters, that is described in detail in Ref. [43].

\section{SUMMARY}

We studied magnetization configurations of soft ferromagnetic dots and films, coupled by the dipolar interaction and, possibly, by interlayer exchange, to a hard matrix magnetized out of plane and having an antidot (circular hole). These nanostructures support the formation of nontrivial magnetization textures-vortices and skyrmions, respectively. Depending on the geometry and material parameters of the patterned films, the radial vortex (Neel skyrmion) or unconventional vortex (skyrmion) can be achieved in the soft magnetic dot (film). The latter unconventional textures are characterized by the position-dependent helicity $\gamma(r)$. Simultaneously, common Bloch vortices or skyrmions cannot be achieved in these nanostructures until the hard matrix produces nonzero radial stray fields, averaged over the dot (film) thickness. These stray fields play a crucial role in the stabilization of nontrivial magnetization textures, as well as in the determination of the helicity of textures.

The averaged helicity of the vortices and skyrmions, stabilized by the stray fields of radial symmetry, gradually changes from Neel-like state $\gamma=0, \pi$ to Bloch-like state $\gamma \rightarrow \pm \pi / 2$ in the limit of negligible stray fields created by the antidot with the variation of geometrical and material parameters. Therefore, the proposed nanostructures with the bias by the stray fields of radial symmetry reveal an additional degree of freedom - the helicity of the vortex (skyrmion), which can be tuned on demand by a proper choice of the nanostructure parameters. The helicity of magnetization texture affects its interaction with external magnetic fields, spin-polarized and pure spin currents, and other dynamical characteristics.

We developed an analytical model to describe the transition from the radial (Neel) state to an unconventional one. The model shows that the Neel-like state is favored by the following factors: (i) a larger ratio of the matrix saturation magnetization to the dot (film) magnetization $M_{\mathrm{HL}} / M_{\mathrm{d}}$, (ii) smaller diameters of the dots (antidots), (iii) an increase of the hard layer thickness, and (iv) a decrease of the dot thickness. All of them are a consequence of the interplay between the Zeeman energy of magnetization in the radial stray fields of the antidot matrix and demagnetization energy of nonuniform magnetization configuration. Additionally, the presence of the exchange coupling with the matrix at the dot lateral edges also increases the range of the radial vortex stability, since it suppresses the penalty of the edge magnetic charge contribution of the demagnetization energy for the Neel-like state. Helicity of the magnetic skyrmions in soft magnetic films coupled to a hard antidot matrix behaves the same as that of vortices in the dots with contact to the matrix, provided by the sufficient interlayer exchange to guarantee the stable out-ofplane magnetization state of the film away from the antidot.

\section{ACKNOWLEDGMENTS}

The Portuguese team acknowledges the Network of Extreme Conditions Laboratories and Portuguese Foundation of Science and Technology for support through Projects No. NORTE-01-0145-FEDER-022096, No. MIT-EXPL/IRA/0012/2017, No. PTDC/FISMAC/31302/2017, No. POCI-0145-FEDER-030085 (NOVAMAG), No. PTDC/FIS-MAC/31302/2017, and No. EXPL/IF/00541/2015 (S.A.B.) and Grant No. SFRH/BPD/90471/2012 (A.H.-R.). Work was also supported by the Ministry of Education and Science of Ukraine, Project No. 0118 U004007 (R.V.V., B.A.I.); IKERBASQUE (the Basque Foundation for Science) (K.Y.G.); the Spanish Ministry of Economy, Industry and Competitiveness, Grant No. FIS2016-78591-C3-3-R (K.Y.G.); the European Union Horizon 2020 Research and Innovation Programme under Marie Skłodowska-Curie Grants No. 644348 (R.V.V., B.A.I., K.Y.G.), No. H2020-MSCA-RISE-2016-734801 (D.N., G.N.K.), and No. H2020-MSCA-IF-2016-74695 (A.H.-R.); the Program of NUST "MISiS," Grant No. K2-2019-006, implemented by Russian Federation government Decree No. 211 dated 16 March 2013 (B.A.I.); the Spanish Ministry for Science, Innovation and Universities, for funding through the "Ramón y Cajal" Program No. RYC-2017-22820 (D.N.); and European Cooperation in Science and Technology, Projects No. CA16218 "NANOCOHYBRI" (G.N.K.) and No. CA17123 “MAGNETOFON” (B.A.I). 


\section{APPENDIX: TOPOLOGICAL CHARACTERISTICS OF VORTICES AND SKYRMIONS}

Here we briefly review topological characteristics of twodimensional magnetization textures, show in which textures the helicity is important, and give a more general definition of the helicity, which is applicable to the textures showing no circular symmetry.

The standard topological analysis is based on the introduction of different topological charges, defined by the mapping of the coordinate space onto the order-parameter space (or its significant subspaces, like degeneracy space $\mathbb{M}_{D}$; see Refs. [19,20]). For ferromagnets, the order-parameter space is the two-dimensional unit sphere of the normalized magnetization $|\boldsymbol{m}|^{2}=1$, where $\boldsymbol{m}=\boldsymbol{M} / M_{s}$. The number of times that the magnetization covers the unit sphere while going along the coordinate space defines the $\pi_{2}$ topological charge $Q$. Mathematically, the topological charge can be calculated as

$$
Q=\frac{1}{4 \pi} \int \boldsymbol{m} \cdot\left(\frac{\partial \boldsymbol{m}}{\partial x} \times \frac{\partial \boldsymbol{m}}{\partial y}\right) d \boldsymbol{r} .
$$

The topological charge $Q$ is naturally characteristic for magnetization textures in easy-axis ferromagnets, in which magnetization is uniform far from the origin. In this case, $Q$ is an integer number. The states with different values of $Q$ cannot be transformed into each other by any local continuous transformation [20,21,59].

Magnetization distribution of skyrmions can be considered as a stereographic projection of the unit sphere onto a two-dimensional plane. In other words, skyrmion distribution covers the unit sphere one time, and, therefore, the topological charge of skyrmions is $Q= \pm 1$. For this reason $Q$ is often called "the skyrmion number." For a uniform magnetization distribution, naturally, $Q=0$, meaning that it is topologically trivial. More complex states with higher topological charge, e.g., biskyrmions having $Q= \pm 2$, have been observed recently in experiments and attract also significant research interest [60-63]. The skyrmion number, of course, can be calculated for any magnetization texture, not obviously having uniform magnetization far from the origin. For example, for common magnetic vortices in easy-plane ferromagnets [shown in Figs. 1(b) and 1(c)] it is equal to $Q= \pm 1 / 2$, i.e., magnetization distribution of a vortex covers only a half of the unit sphere $|\boldsymbol{m}|^{2}=1$.

The standard vortices in all ordered media are defined as topological textures with nonuniform order parameter far from the origin (formally, at $r \rightarrow \infty$ ). Such structures can be realized in systems with continuous degeneracy, when the ground state corresponds to the values of the order parameter from the degeneracy space $\mathbb{M}_{D}$. For example, in an easy-plane ferromagnet (e.g., ferromagnetic film, which demonstrates natural easy-plane shape anisotropy) all in-plane magnetization directions are degenerate, so the degeneracy space $\mathbb{M}_{\mathrm{D}}$ is the circle $m_{z}=0$ at the unit sphere $|\boldsymbol{m}|^{2}=1$. The classification of vortices is based on the mapping of the order-parameter distribution along a closed contour in the coordinate space into the degeneracy space $\mathbb{M}_{D}$. This mapping is described by the topological charge, the values of which are elements of the so-called fundamental homotopy group $\pi_{1}$ of $\mathbb{M}_{\mathrm{D}}$. In general, the mathematical properties of these groups can be quite complicated [19,20], but in the case of ferromagnetic vortices they are much simplified. In this case the $\pi_{1}$ topological charge just defines how many times magnetization rotates in the easy plane $\mathbb{M}_{D}$ when passing a closed contour far from the vortex core. The $\pi_{1}$ topological charge can be calculated as $[20,21,64]$

$$
v=\frac{1}{2 \pi} \oint_{l_{z}=0} \boldsymbol{e}_{z} \cdot(\boldsymbol{l} \times d \boldsymbol{l})=\frac{1}{2 \pi} \int \frac{\partial \phi}{\partial \chi} d \chi,
$$

where $\boldsymbol{l}$ is the unit vector tangential to the integration path and $\boldsymbol{e}_{z}$ is the unit vector along the hard axis of the magnet (the polar axis). The quantity $v$ is called "the winding number," or "vorticity." Naturally, vorticity is an integer number, $v \in \mathbb{Z}$, as required by the continuity of magnetization distribution. The definition of vorticity Eq. (A2) can be also applied to skyrmions and other two-dimensional textures as well. For simple symmetric structures the vorticity appears in the simple relation between polar magnetization and polar coordinate angles, $\phi=v \chi+\gamma$. Vortices and skyrmions are characterized by the vorticity $v=+1$; uniform magnetization distribution has, naturally, $v=0$. Winding number $v=-1$ is realized, for instance, in other interesting topological objectsantivortices $[65,66]$ and antiskyrmions $[67,68]$. Textures with higher winding numbers, $|v| \geqslant 2$, can be easily imagined, but we are not aware of their experimental realization. Also, we would like to note that the vorticity can be nonzero for nontopological textures having topological charge $Q=0$. For example, the nontopological counterpart of the skyrmion (a "skyrmion with reversed core"), which can be also stabilized by dipolar stray fields [44], has $v=+1$ and $Q=0$.

Now let us discuss in more detail the last characteristic of a two-dimensional topological texture, the parameter $\gamma$, which can be considered as an initial angle of in-plane magnetization, $\phi=v \chi+\gamma$. It should be noted that this parameter has a principal sense only for textures with the winding number $v=+1$. Indeed, by rotating the coordinate system at an angle $\psi\left(\chi=\chi^{\prime}+\psi, \phi=\phi^{\prime}+\psi\right)$, the parameter $\gamma$ changes if $v \neq 1$. Moreover, choosing $\psi=\gamma /(1-v)$ one gets the relation $\phi^{\prime}=v \chi^{\prime}$. Thus, for $v \neq 1$, the parameter $\gamma$ defines the rotation of the magnetization texture in its plane as whole, but not the internal structure of a texture, and can be eliminated by a proper choice of the coordinate system.

The only exceptions are textures with the winding number $v=+1$, in which $\gamma$ cannot be eliminated and has a sense of the helicity of the magnetization texture. The geometrical meaning of the helicity in this case is the angle between the radial direction and the in-plane magnetization component [Fig. 1(a)]. In simple magnetization textures this angle is constant, and we can define the helicity simply as

$$
\gamma=\arcsin \frac{m_{\chi}}{m_{\mathrm{IP}}}
$$

where $m_{\mathrm{IP}}=\sqrt{m_{r}^{2}+m_{\chi}^{2}}$ is the in-plane component of magnetization. This case stands, for example, for Bloch and Neel skyrmions and vortices, shown in Figs. 1(b)-1(e). In a more general case, however, the angle between radial direction and the in-plane magnetization component can be coordinate dependent, and the helicity, defined by the equation above, becomes also coordinate dependent, $\gamma=\gamma(r, \chi)$. In this more 
general case we can introduce the averaged helicity of the texture:

$$
\bar{\gamma}=\arcsin \left[\frac{\int m_{\chi} d \boldsymbol{r}}{\int m_{\mathrm{IP}} d \boldsymbol{r}}\right]=\arcsin \left[\frac{\int \sin (\phi-\chi) \sin \theta d \boldsymbol{r}}{\int \sin \theta d \boldsymbol{r}}\right] .
$$

This definition is used in Sec. IV of the main text. An alternative way to describe the helicity of the topological magnetization textures was used in Ref. [69], where $\gamma$ was defined at $r=r_{s}$, where $r_{s}$ is the vortex/skyrmion radius.
[1] Skyrmions: Topological Structures, Properties, and Applications, edited by J. Liu, Z. Zhang, and G. Zhao, Series in Materials Science and Engineering (CRC, Boca Raton, FL, 2016).

[2] G. Finocchio, F. Büttner, R. Tomasello, M. Carpentieri, and M. Kläui, J. Phys. D 49, 423001 (2016).

[3] A. Fert, N. Reyren, and V. Cros, Nat. Rev. Mater. 2, 17031 (2017).

[4] K. Y. Guslienko, J. Nanosci. Nanotechnol. 8, 2745 (2008).

[5] R. Antos, Y. Otani, and J. Shibata, J. Phys. Soc. Jpn. 77, 031004 (2008).

[6] A. Thiaville, S. Rohart, É. Jué, V. Cros, and A. Fert, Europhys. Lett. 100, 57002 (2012).

[7] K.-S. Ryu, L. Thomas, S.-H. Yang, and S. Parkin, Nature Nanotech. 8, 527 (2013).

[8] F. Zheng, F. N. Rybakov, A. B. Borisov, D. Song, S. Wang, Z.-A. Li, H. Du, N. S. Kiselev, J. Caron, A. Kovács, M. Tian, Y. Zhang, S. Blügel, and R. E. Dunin-Borkowski, Nature Nanotech. 13, 451 (2018).

[9] W. Döring, J. Appl. Phys. 39, 1006 (1968).

[10] E. Galkina, B. Ivanov, and V. Stephanovich, J. Magn. Magn. Mater. 118, 373 (1993).

[11] C. Andreas, A. Kákay, and R. Hertel, Phys. Rev. B 89, 134403 (2014).

[12] I. E. Dzyloshinskiǐ and B. A. Ivanov, JETP Lett. 29, 540 (1979).

[13] A. B. Borisov and F. N. Rybakov, JETP Lett. 88, 264 (2008).

[14] A. Fert, V. Cros, and J. Sampaio, Nature Nanotech. 8, 152 (2013).

[15] N. Romming, C. Hanneken, M. Menzel, J. E. Bickel, B. Wolter, K. von Bergmann, A. Kubetzka, and R. Wiesendanger, Science 341, 636 (2013).

[16] A. Dussaux, B. Georges, J. Grollier, V. Cros, A. V. Khvalkovskiy, A. Fukushima, M. Konoto, H. Kubota, K. Yakushiji, S. Yuasa, K. A. Zvezdin, K. Ando, and A. Fert, Nature Commun. 1, 8 (2010).

[17] S. Tsunegi, H. Kubota, K. Yakushiji, M. Konoto, S. Tamaru, A. Fukushima, H. Arai, H. Imamura, E. Grimaldi, R. Lebrun, J. Grollier, V. Cros, and S. Yuasa, Appl. Phys. Express 7, 063009 (2014).

[18] Y. Li, X. de Milly, O. Klein, V. Cros, J. Grollier, and G. de Loubens, Appl. Phys. Lett. 112, 022405 (2018).

[19] G. E. Volovik and V. P. Mineev, Sov. Phys. JETP 45, 1186 (1977).

[20] N. D. Mermin, Rev. Mod. Phys. 51, 591 (1979).

[21] H.-B. Braun, Adv. Phys. 61, 1 (2012).

[22] N. Nagaosa and Y. Tokura, Nature Nanotech. 8, 899 (2013).

[23] N. Usov and S. Peschany, J. Magn. Magn. Mater. 118, L290 (1993).
[24] G. Siracusano, R. Tomasello, A. Giordano, V. Puliafito, B. Azzerboni, O. Ozatay, M. Carpentieri, and G. Finocchio, Phys. Rev. Lett. 117, 087204 (2016).

[25] V. Karakas, A. Gokce, A. T. Habiboglu, S. Arpaci, K. Ozbozduman, I. Cinar, C. Yanik, R. Tomasello, S. Tacchi, G. Siracusano, M. Carpentieri, G. Finocchio, T. Hauet, and O. Ozatay, Sci. Rep. 8, 7180 (2018).

[26] C. Phatak, A. K. Petford-Long, and O. Heinonen, Phys. Rev. Lett. 108, 067205 (2012).

[27] S. Wintz, C. Bunce, A. Neudert, M. Körner, T. Strache, M. Buhl, A. Erbe, S. Gemming, J. Raabe, C. Quitmann, and J. Fassbender, Phys. Rev. Lett. 110, 177201 (2013).

[28] M. Yan, H. Wang, and C. Campbell, J. Magn. Magn. Mater. 320, 1937 (2008).

[29] K. Y. Guslienko, G. N. Kakazei, J. Ding, X. M. Liu, and A. O. Adeyeye, Sci. Rep. 5, 13881 (2015).

[30] R. V. Verba, A. Hierro-Rodriguez, D. Navas, J. Ding, X. M. Liu, A. O. Adeyeye, K. Y. Guslienko, and G. N. Kakazei, Phys. Rev. B 93, 214437 (2016).

[31] J. Sampaio, V. Cros, S. Rohart, A. Thiaville, and A. Fert, Nature Nanotech. 8, 839 (2013).

[32] S. D. Pollard, J. A. Garlow, J. Yu, Z. Wang, Y. Zhu, and H. Yang, Nature Commun. 8, 14761 (2017).

[33] O. Boulle, J. Vogel, H. Yang, S. Pizzini, d. S. C. Dayane, A. Locatelli, T. O. Menteş, A. Sala, L. D. Buda-Prejbeanu, O. Klein, M. Belmeguenai, Y. Roussigné, A. Stashkevich, S. M. Chérif, L. Aballe, M. Foerster, M. Chshiev, S. Auffret, I. M. Miron, and G. Gaudin, Nat. Nanotech. 11, 449 (2016).

[34] K. Shibata, X. Z. Yu, T. Hara, D. Morikawa, N. Kanazawa, K. Kimoto, S. Ishiwata, Y. Matsui, and Y. Tokura, Nature Nanotech. 8, 723 (2013).

[35] K. Karube, K. Shibata, J. S. White, T. Koretsune, X. Z. Yu, Y. Tokunaga, H. M. Rønnow, R. Arita, T. Arima, Y. Tokura, and Y. Taguchi, Phys. Rev. B 98, 155120 (2018).

[36] L. Sun, R. X. Cao, B. F. Miao, Z. Feng, B. You, D. Wu, W. Zhang, A. Hu, and H. F. Ding, Phys. Rev. Lett. 110, 167201 (2013).

[37] K. Y. Guslienko, IEEE Magn. Lett. 6, 4000104 (2015).

[38] A. S. Ahmed, B. D. Esser, J. Rowland, D. W. McComb, and R. K. Kawakami, J. Cryst. Growth 467, 38 (2017).

[39] Y. Dovzhenko, F. Casola, S. Schlotter, T. X. Zhou, F. Büttner, R. L. Walsworth, G. S. D. Beach, and A. Yacoby, Nat. Commun. 9, 2712 (2018).

[40] W. Legrand, J.-Y. Chauleau, D. Maccariello, N. Reyren, S. Collin, K. Bouzehouane, N. Jaouen, V. Cros, and A. Fert, Sci. Adv. 4, eaat0415 (2018).

[41] W. Legrand, N. Ronceray, N. Reyren, D. Maccariello, V. Cros, and A. Fert, Phys. Rev. Appl. 10, 064042 (2018). 
[42] T. Srivastava, M. Schott, R. Juge, V. Křižáková, M. Belmeguenai, Y. Roussigné, A. Bernand-Mantel, L. Ranno, S. Pizzini, S.-M. Chérif, A. Stashkevich, S. Auffret, O. Boulle, G. Gaudin, M. Chshiev, C. Baraduc, and H. Béa, Nano Lett. 18, 4871 (2018).

[43] R. V. Verba, D. Navas, A. Hierro-Rodriguez, S. A. Bunyaev, B. A. Ivanov, K. Y. Guslienko, and G. N. Kakazei, Phys. Rev. Appl. 10, 031002 (2018).

[44] D. Navas, R. V. Verba, A. Hierro-Rodriguez, S. A. Bunyaev, X. Zhou, A. O. Adeyeye, O. V. Dobrovolskiy, B. A. Ivanov, K. Y. Guslienko, and G. N. Kakazei, APL Mater. 7, 081114 (2019).

[45] E. Berganza, M. Jaafar, M. Goiriena-Goikoetxea, J. PabloNavarro, A. Garcia-Arribas, K. Gusliyenko, C. Magen, J. M. de Teresa, O. Chubykalo-Fesenko, and A. Asenjo, arXiv:1803.08768 (2018).

[46] S. Zhang, J. Zhang, Q. Zhang, C. Barton, V. Neu, Y. Zhao, Z. Hou, Y. Wen, C. Gong, O. Kazakova, W. Wang, Y. Peng, D. A. Garanin, E. M. Chudnovsky, and X. Zhang, Appl. Phys. Lett. 112, 132405 (2018).

[47] D. A. Garanin, D. Capic, S. Zhang, X. Zhang, and E. M. Chudnovsky, J. Appl. Phys. 124, 113901 (2018).

[48] A. Vansteenkiste, J. Leliaert, M. Dvornik, M. Helsen, F. GarciaSanchez, and B. Van Waeyenberge, AIP Advances 4, 107133 (2014).

[49] R. F. C. Farrow, D. Weller, R. F. Marks, M. F. Toney, D. J. Smith, and M. R. McCartney, J. Appl. Phys. 84, 934 (1998).

[50] S. Dhesi, G. van der Laan, H. Dürr, M. Belakhovsky, S. Marchesini, P. Kamp, A. Marty, B. Gilles, and A. Rogalev, J. Magn. Magn. Mater. 226-230, 1580 (2001).

[51] J. Gräfe, M. Weigand, N. Träger, G. Schütz, E. J. Goering, M. Skripnik, U. Nowak, F. Haering, P. Ziemann, and U. Wiedwald, Phys. Rev. B 93, 104421 (2016).

[52] M. Krupinski, D. Mitin, A. Zarzycki, A. Szkudlarek, M. Giersig, M. Albrecht, and M. Marszałek, Nanotechnology 28, 085302 (2017).
[53] S. A. Díaz and R. E. Troncoso, J. Phys.: Cond. Matter 28, 426005 (2016).

[54] F. Garcia-Sanchez, J. Sampaio, N. Reyren, V. Cros, and J.-V. Kim, New J. Phys. 18, 075011 (2016).

[55] A. Y. Galkin and B. A. Ivanov, JETP 109, 74 (2009).

[56] K. Y. Guslienko and A. N. Slavin, J. Magn. Magn. Mater. 323, 2418 (2011).

[57] K. Y. Guslienko and A. N. Slavin, J. Appl. Phys. 87, 6337 (2000).

[58] L. E. Elsgolc, Calculus of Variations (Pergamon, New York, 1961).

[59] A. Kosevich, B. Ivanov, and A. Kovalev, Phys. Rep. 194, 117 (1990).

[60] X. Z. Yu, Y. Tokunaga, Y. Kaneko, W. Z. Zhang, K. Kimoto, Y. Matsui, Y. Taguchi, and Y. Tokura, Nat. Commun. 5, 3198 (2014).

[61] W. Wang, Y. Zhang, G. Xu, L. Peng, B. Ding, Y. Wang, Z. Hou, X. Zhang, X. Li, E. Liu, S. Wang, J. Cai, F. Wang, J. Li, F. $\mathrm{Hu}, \mathrm{G}$. Wu, B. Shen, and X.-X. Zhang, Adv. Mater. 28, 6887 (2016).

[62] E. G. Galkina, E. V. Kirichenko, B. A. Ivanov, and V. A. Stephanovich, Phys. Rev. B 79, 134439 (2009).

[63] D. Capic, D. A. Garanin, and E. M. Chudnovsky, Phys. Rev. B 100, 014432 (2019).

[64] B. A. Ivanov, Low Temp. Phys. 31, 635 (2005).

[65] H. Wang and C. E. Campbell, Phys. Rev. B 76, 220407(R) (2007).

[66] A. Haldar and K. S. Buchanan, Appl. Phys. Lett. 102, 112401 (2013).

[67] W. Koshibae and N. Nagaosa, Nat. Commun. 7, 10542 (2016).

[68] A. K. Nayak, V. Kumar, T. Ma, P. Werner, E. Pippel, R. Sahoo, F. Damay, U. K. Rößler, C. Felser, and S. S. P. Parkin, Nature (London) 548, 561 (2017).

[69] M. Mruczkiewicz, M. Krawczyk, and K. Y. Guslienko, Phys. Rev. B 95, 094414 (2017). 